International Journal of

Technology Assessment in

Health Care

cambridge.org/thc

\section{Policy}

Cite this article: Eichler H-G et al (2021). Exploring the opportunities for alignment of regulatory postauthorization requirements and data required for performance-based managed entry agreements. International Journal of Technology Assessment in Health Care 37, e83, 1-11. https://doi.org/10.1017/ S026646232100057X

Received: 3 March 2021

Revised: 12 July 2021

Accepted: 30 July 2021

\section{Key words:}

Pricing and reimbursement; Managed entry agreement; Real-world data; Drug regulation

Author for correspondence:

Hans-Georg Eichler,

E-mail: hgeichler@gmx.net (c) The Author(s), 2021. Published by Cambridge University Press. This is an Open Access article, distributed under the terms of the Creative Commons Attribution licence (http://creativecommons.org/licenses/by/4.0/), which permits unrestricted re-use, distribution and reproduction, provided the original article is properly cited.

\section{CAMBRIDGE} UNIVERSITY PRESS

\title{
Exploring the opportunities for alignment of regulatory postauthorization requirements and data required for performance-based managed entry agreements
}

Hans-Georg Eichler ${ }^{1,2}$ (D), Roisin Adams ${ }^{3,4}$, Einar Andreassen ${ }^{5}$, Peter Arlett ${ }^{6,7}$, Marc van de Casteele $^{8}$, Suzannah J. Chapman ${ }^{9}$, Wim G. Goettsch ${ }^{10,11}$ (D), Jonathan Lind Martinsson ${ }^{12}$, Jordi Llinares-Garcia ${ }^{1}$, Anna Nachtnebel ${ }^{13}$, Elias Pean ${ }^{14}$, Guido Rasi ${ }^{15,16}$, Tove Ragna Reksten ${ }^{5}$, Lonneke Timmers ${ }^{10}$, Rick A. Vreman ${ }^{10,11}$ (D), Inneke van de Vijver $^{8}$ and Martin Wenzl ${ }^{9}$

\footnotetext{
${ }^{1}$ Regulatory Science and Innovation Task Force, European Medicines Agency (EMA), Amsterdam, The Netherlands; ${ }^{2}$ Medical University of Vienna, Vienna, Austria; ${ }^{3}$ National Centre for Pharmacoeconomics (NCPE Ireland), Dublin, Ireland; ${ }^{4}$ Trinity College Dublin, Dublin, Ireland; ${ }^{5}$ Statens Legemiddelverk (SLV), Oslo, Norway; ${ }^{6}$ Data Analytics and Methods Task Force, European Medicines Agency (EMA), Amsterdam, The Netherlands; ${ }^{7}$ London School of Hygiene and Tropical Medicine, London, UK; ${ }^{8}$ Department of Pharmaceutical Policy, National Institute for Health and Disability Insurance (NIHDI/RIZIV/INAMI), Brussels, Belgium; ${ }^{9}$ Organisation for Economic Co-operation and Development (OECD), Paris, France; ${ }^{10}$ National Health Care Institute (ZIN), Diemen, The Netherlands; ${ }^{11}$ Division of Pharmacoepidemiology \& Clinical Pharmacology, Utrecht Institute for Pharmaceutical Sciences (UIPS), Utrecht University, Utrecht, The Netherlands; ${ }^{12}$ Department of Value Based Pricing, Tandvårds- och läkemedelsförmånsverket (TLV), Stockholm, Sweden; ${ }^{13}$ Dachverband der Sozialversicherungsträger (DVSV), Vienna, Austria; ${ }^{14}$ Office of Oncology and Haematology, European Medicines Agency (EMA), Amsterdam, The Netherlands; ${ }^{15}$ European Medicines Agency, Amsterdam, The Netherlands and ${ }^{16}$ Department of Experimental Medicine, Universita di Tor Vergata, Rome, Italy
}

\begin{abstract}
Performance-based managed entry agreements (PB-MEAs) might allow patient access to new medicines, but practical hurdles make competent authorities for pricing and reimbursement (CAPR) reluctant to implement PB-MEAs. We explored if the feasibility of PB-MEAs might improve by better aligning regulatory postauthorization requirements with the data generation of PB-MEAs and by active collaboration and data sharing. Reviewers from seven CAPRs provided structured assessments of the information available at the European Medicines Agency (EMA) Web site on regulatory postauthorization requirements for fifteen recently authorized products. The reviewers judged to what extent regulatory postauthorization studies could help implement PBMEAs by addressing uncertainty gaps. Study domains assessed were: patient population, intervention, comparators, outcomes, time horizon, anticipated data quality, and anticipated robustness of analysis. Reviewers shared general comments about PB-MEAs for each product and on cooperation with other CAPRs. Reviewers rated regulatory postauthorization requirements at least partly helpful for most products and across domains except the comparator domain. One quarter of responses indicated that public information provided by the EMA was insufficient to support the implementation of PBMEAs. Few PB-MEAs were in place for these products, but the potential for implementation of PB-MEAs or collaboration across CAPRs was seen as more favorable. Responses helped delineate a set of conditions where PB-MEAs may help reduce uncertainty. In conclusion, PB-MEAs are not a preferred option for CAPRs, but we identified conditions where PB-MEAs might be worth considering. The complexities of implementing PB-MEAs remain a hurdle, but collaboration across silos and more transparency on postauthorization studies could help overcome some barriers.
\end{abstract}

\section{Introduction}

New treatment options may improve patients' lives, but the growing complexity of some new health technologies (e.g., gene and cell therapies) that come with considerable uncertainties about their clinical benefit and at high prices is becoming a barrier for patient access.

Competent authorities for pricing and reimbursement (CAPR), which include healthcare payers and, in some jurisdictions, health technology assessment (HTA) agencies, want to make efficient, well-informed decisions without delaying patient access. However, they are wary of the consequences of making a less optimal coverage decision in the context of 
uncertain effects of a new treatment on health outcomes and/or healthcare budgets (1). Performance-based managed entry agreements (PB-MEAs, also widely known as performance-based agreements, PBAs) have been proposed as a means to address these barriers (2) with the objectives of progressively reducing uncertainties about a drug's performance, mitigating healthcare payers' financial risk, and managing budget impact.

Conceptually, PB-MEAs could help assuage payers' concerns, while facilitating patient access by paying firms only for treatments to which patients respond (patient level), by making payments to firms contingent on the results achieved at the population level, or by coupling temporary coverage with further evidence development. All three of these arrangements require patient clinical measures to be collected and linked to financial consequences, based on a preagreed plan (2). However, the track record of PB-MEAs implemented to date in several countries of the Organisation for Economic Co-operation and Development (OECD) is mixed; critics argue that PB-MEAs increase administrative burden, reduce transparency, and that anticipated results are often not forthcoming or difficult to interpret (2).

Regulatory agencies assess new treatments' benefits and harms to the exclusion of economic considerations, whereas payers focus on effectiveness and economic consequences. Yet, both types of decision makers usually see the same sets of clinical data and may request drug manufacturers to generate new clinical data. We hypothesized that the effectiveness of PB-MEAs in achieving their stated objectives can be increased by (i) better alignment of research questions, data sources, and analytic methods between the postauthorization requirements requested by regulators around the time of marketing authorization and the data generation of PB-MEAs and (ii) by making available the clinical results of PB-MEAs to other decision makers.

Even though PB-MEAs need to be customized to therapies and circumstances, we hypothesized that the clinical findings from PB-MEAs-if shared with regulators-could facilitate postauthorization monitoring of drug safety and effectiveness and contribute additional knowledge about, for example, benefit-risk assessment in defined patient subgroups, optimal position of a drug in treatment pathways, or even provide a more accurate prediction of its cost-effectiveness. Sharing of such data across decision makers can also support pricing and reimbursement decisions in jurisdictions beyond the one where the data were collected and ultimately facilitate patient access.

A reasonable first step in exploring the opportunities and obstacles of regulator-CAPR collaboration would be to assess what is already available. At the time of marketing authorization, regulatory agencies, like the European Medicines Agency (EMA), discuss and agree with manufacturers a plan for postauthorization evidence generation. For the EU, the result of such agreements is made public by the EMA and might help CAPRs in accepting and designing a PB-MEA for the product.

We, therefore, conducted a survey, based on a sample of products recently authorized in the $\mathrm{EU}$, with a view to

(1) gaining insights into the reasons why, in the views of CAPRs, a PB-MEA might be beneficial in terms of addressing the uncertainty gaps and providing a framework for risk-sharing financial agreements, supporting a well-informed coverage decision, and what conditions need to be in place for a given product-indication pair for a PB-MEA to fulfill its objectives;
(2) estimating how much of the information gleaned from regulatory postauthorization requirements could have been used to support implementing PB-MEAs;

(3) defining what additional information would have been needed to inform a PB-MEA;

(4) outlining what further concrete steps could be taken by CAPRs and regulators to improve the effectiveness of PB-MEAs in achieving their stated goals and to support regulators in delivering on their mission to monitor drug performance;

(5) helping define a possible framework for collaboration and information sharing across CAPR and between regulators and CAPR, while respecting their respective roles and remits.

We, here, report results from our analysis and propose future activities to realize potential synergies between regulatory postauthorization requirements and the establishment of PB-MEAs.

\section{Methods}

The analysis was informed by a recent OECD report on the experiences with PB-MEAs in several OECD countries (2). For the purposes of our study, we adopted the OECD report's definition of PB-MEAs; the report provides a detailed taxonomy of the types of PB-MEAs.

All study team members developed and agreed upon an assessment template with a set of questions to give feedback on each individual product-indication pair from a predetermined list (see below).

Study team members from the EMA provided reviewers from selected CAPRs with user-friendly links to the individual products' European Public Assessment Report (EPAR) on the EMA Web site that contains information on postauthorization requirements.

Study teams from the individual CAPRs assessed the information available at the EMA Web site and provided their aggregated feedback. Reviewers from CAPR organizations were assisted by EMA staff to answer questions for clarification.

\section{Participating CAPRs}

The seven participating CAPRs were identified through informal exploration of their willingness to contribute, thus representing a convenience sample:

Australia: Technology Assessment and Access Division, Commonwealth Department of Health, Austria: Dachverband der Sozialversicherungsträger (DVSV), Belgium: National Institute for Health and Disability Insurance (NIHDI/RIZIV/INAMI), Ireland: National Centre for Pharmacoeconomics (NCPE), the Netherlands: National Health Care Institute (ZIN), Norway: Statens legemiddelverk (SLV), Sweden: Tandvårds- och läkemedelsförmånsverket (TLV). Reviewers from CAPRs were employees of their respective organizations and experienced in drug evaluation for reimbursement.

Representation from drug regulatory agencies came from the EMA.

\section{Study Sample (Product-Indication Pairs)}

The study sample, shown in Table 1, was based on the sample of product-indication pairs used by the OECD in its recent report on PB-MEAs (2). It was agreed that this list would be ranked by date of authorization in the EU, identifying the fifteen product-indication pairs most recently authorized and excluding multiple indications for a given product, that is, if there was more 
Table 1. Study sample

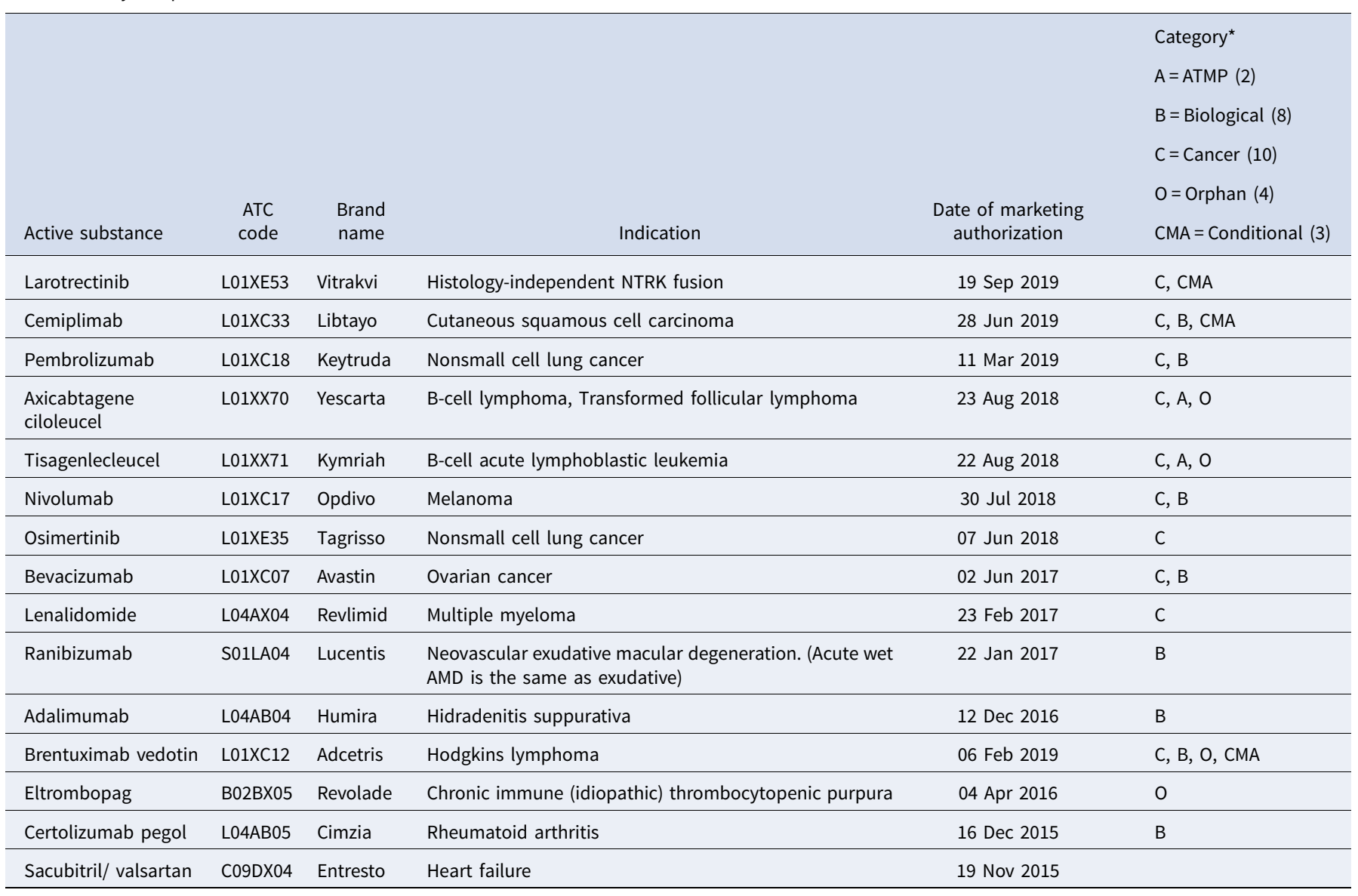

Fifteen product-indication pairs were selected for the survey. For selection criteria, refer to the "Methods" section.

ATMP, Advanced Therapy Medicinal Product (comprises cell, gene, and modified tissue therapies); CMA, conditional marketing authorization.

than one indication per product, only the most recently authorized product-indication pair was selected. It was expected that this study cohort would include a substantial number of cancer and orphan product-indication pairs, and all of them would have postauthorization requirements. It was agreed that if this list did not include at least three conditionally authorized products (3), the products lowest on the list (i.e., the oldest) would be replaced by recently conditionally authorized products to ensure that at least three conditionally authorized products are included in the final list of fifteen product-indication pairs.

\section{Scope of Analysis and Classification of Findings}

The scope of the analysis performed by CAPR reviewers aimed to reflect the key elements of a $\mathrm{PB}-\mathrm{MEA}$ required to achieve its stated goals related to performance of the treatment. It was based on previous evaluations of regulator-CAPR collaborations (4) and comprised the following domains:

(1) patient population (including subgroup information);

(2) intervention (relevance of dose, regimen, place in therapeutic pathway, setting in the healthcare system);

(3) comparators (ability to draw conclusions about comparative effectiveness, direct or indirect analyses);

(4) outcomes (relevance of end point(s) to patients and CAPRs);

(5) time horizon (duration of the observation period);
(6) anticipated data quality;

(7) anticipated robustness of analysis.

All other elements of such agreements, including financial, not related to the performance of the treatment were excluded. The CAPR reviewers were asked to assess, for each product-indication pair, the postauthorization requirements formulated by the EMA and to answer the question, "Are the post-authorization requirements related to this parameter likely appropriate to reduce uncertainty about the drug's performance and to design and execute a successful PB-MEA?" The reviewers were asked to categorize each parameter as either:

(1) likely appropriate/relevant as is;

(2) partly appropriate/relevant (provide comments on what other information/methodology would be needed);

(3) inadequate/inappropriate/not relevant (provide reasons);

(4) insufficient information available, specify what additional information is needed or not applicable.

In addition, the reviewers were asked:

(1) To explain if a PB-MEA for the specific product-indication had been put in place or if not, what the barriers would have been. Would a PB-MEA have been preferred if these barriers were overcome? Why? 
(2) If cooperation with other CAPRs would help address the uncertainties that warrant a PB-MEA and, if yes, what kind of cooperation.

(3) To provide additional reflections on each parameter (optional).

We analyzed results from the survey in quantitative and qualitative ways. Quantitative analysis was limited to counts of responses by CAPR reviewers across the categories in the survey (described above and shown in Tables 2 and 3).

For qualitative analysis, we extracted free text responses that were deemed to have generic relevance, excluding those that were highly specific to one product-indication pair only. Hence, the statements listed represent archetypes of issues, barriers, and opportunities perceived by CAPRs. Where applicable, responses to individual questions are grouped by overarching themes (Table 4).

Reviewers (the number of reviewers per CAPR: 2-3) from each of the seven individual CAPRs were asked to aggregate their comments and responses in one separate, preagreed assessment template for each product-indication pair $(n=15)$. This was done to, on the one hand, draw from a broader knowledge base and, on the other hand, to obtain a unique response "per organization," that is at the level where decisions are taken, rather than "per person." For Australia, only publicly available information from Public Summary Documents (PSDs) was used (5).

\section{Results}

Table 1 shows the final list of fifteen product-indication pairs. All of them had received marketing authorization in the EU, though not necessarily in jurisdictions outside the EU; all of them had some form of postauthorization requirements. Of the fifteen products, two were Advanced Therapy Medicinal Products (ATMPs; comprising cell, gene, and modified tissue therapies, as defined by EU legislation); eight were biologics, ten and four were cancer and orphan product-indication pairs, respectively, and three had received conditional marketing authorization (CMA). Note that regulatory approval does not necessarily imply that a given product is reimbursed or even considered for reimbursement by an individual CAPR (see below and Table 4, part 2).

All seven participating CAPRs provided responses. For nine product-indication pairs, one CAPR was unable to share information specifically on the question whether a PB-MEA is in place; for one additional product-indication pair, two CAPRs were unable to share that information. The inability to provide this information was due to confidentiality agreements being in place. Responses to the other questions were not constrained by confidentiality considerations.

A large proportion of responses were hypothetical, based on the CAPR assessors' general experience with pricing and reimbursement negotiations, rather than reflecting de facto experience with PB-MEAs for the products in question (see Table 2 and below).

\section{Quantitative Analyses}

For the quantitative analyses presented in Tables 2 and 3, responses from different reviewers per CAPR were aggregated, that is each of the seven CAPRs was counted as one response. Responses from the survey on the extent to which regulatory postauthorization requirements could potentially support implementation of a PB-MEA for the fifteen product-indication pairs are summarized in Table 2, upper panel.

The CAPR reviewers rated regulatory postauthorization requirements as either "likely appropriate and relevant as is" or "partly appropriate and relevant" for the majority of productindication pairs and across domains (Table 2, upper panel, column 5), with the exception of the comparators domain, which was frequently deemed "inadequate/inappropriate/not relevant."

However, there was a high number of responses in the category "not applicable or not assessed" (around one quarter of responses, Table 2, upper panel, column 6). Responses in this category were usually based on reasons like "Insufficient information provided by EMA in the public domain" (see Table 4 and Discussion).

Responses to questions on the actual or potential implementation of a PB-MEA or potential added value of collaboration are summarized in Table 2, lower panel. Only one CAPR reviewer reported the implementation of a PB-MEA for one product-indication pair in their jurisdiction; in two further instances, the reviewers indicated that "some aspects of a PB-MEA" were in place.

Yet, the potential benefit of implementing a PB-MEA or collaboration with other CAPRs was seen more favorable (column 5). However, this must be seen, again, in light of the very high number of cases deemed "not applicable or not assessed" (column 6).

Responses grouped for individual product-indication pairs are shown in Table 3.

The numbers of favorable responses, as defined in Table 3 , across all domains of the regulatory postauthorization requirements varied widely between product-indication pairs, with the difference in the numbers of favorable responses between the highest and the lowest-ranked product-indication pairs being more than two-fold (range: 41-19; Table 3, upper panel).

The range of favorable responses to questions about the potential benefits of implementing a PB-MEA and of collaboration with other CAPRs was even wider (Table 3, lower panel): for two product-indication pairs, reviewers from six out of seven CAPRs deemed that collaboration could potentially support the implementation of PB-MEAs, whereas, for other products, there were no favorable responses. The reasons for this high level of heterogeneity are addressed in the qualitative analysis.

\section{Qualitative Analysis}

Detailed reasons given by reviewers from the seven CAPRs for their assessments are listed in Table 4.

The reasons stated why the individual domains of the EMA's postauthorization requirements were deemed inadequate/inappropriate/not relevant are summarized in Table 4, part 1; these related to various aspects of the postauthorization study designs, such as a nonrandomized design, a lack of information on the quality of life, or observation deemed too short.

Answers about the implementation, desirability of a PB-MEA, and cooperation with other CAPRs are listed in Table 4, part 2. There was a wide range of reasons given why a PB-MEA is not in place/not preferred for a given product, including issues of scope and remit of the participating CAPR, the nature of the problem on hand (clinical uncertainty vs financial uncertainty or budget impact) that may dictate other types of agreements, and the complexities in executing PB-MEAs.

The reasons stated why cooperation with other CAPRs would have helped address the uncertainties that warrant a PB-MEA were also highly diverse and went beyond a simple sharing of clinical outcome data, including motivations like jointly incentivizing 
Table 2. Summary of responses to individual survey questions

\begin{tabular}{|c|c|c|c|c|c|}
\hline 1 & 2 & 3 & 4 & 5 & 6 \\
\hline \multirow[t]{2}{*}{$\begin{array}{l}7 \text { respondents }{ }^{*} 15 \text { products } \\
\text { Total } N=105\end{array}$} & $\begin{array}{l}\text { Likely appropriate } \\
\text { and relevant as is }\end{array}$ & $\begin{array}{l}\text { Partly appropriate } \\
\text { and relevant }\end{array}$ & $\begin{array}{l}\text { Inadequate/ } \\
\text { inappropriate/ } \\
\text { not relevant }\end{array}$ & $\begin{array}{l}N \text { and percentage of } \\
\text { responses at least } \\
\text { partly appropriate }\end{array}$ & $\begin{array}{l}\text { Not applicable or } \\
\text { not assessed }\end{array}$ \\
\hline & $N$ & $N$ & $N$ & $N / N(\%)$ & $N / N(\%)$ \\
\hline $\begin{array}{l}\text { Patient population (including subgroup } \\
\text { information) }\end{array}$ & 54 & 17 & 7 & $71 / 78(91.0 \%)$ & $27 / 105(25.7 \%)$ \\
\hline $\begin{array}{l}\text { Intervention (relevance of dose, regimen, } \\
\text { place in therapeutic pathway, setting in } \\
\text { the healthcare system) }\end{array}$ & 52 & 18 & 9 & $70 / 79(88.6 \%)$ & $26 / 105(24.8 \%)$ \\
\hline $\begin{array}{l}\text { Comparators (the ability to draw } \\
\text { conclusions about comparative } \\
\text { effectiveness, direct or indirect analyses) }\end{array}$ & 35 & 13 & 28 & $48 / 76(63.2 \%)$ & $29 / 105(27.6 \%)$ \\
\hline $\begin{array}{l}\text { Outcomes (relevance of end point(s) to } \\
\text { patients, CA PR) }\end{array}$ & 41 & 26 & 11 & $67 / 78(85.9 \%)$ & $27 / 105(25.7 \%)$ \\
\hline $\begin{array}{l}\text { Time horizon (duration of the } \\
\text { observation period) }\end{array}$ & 46 & 20 & 10 & $66 / 76(86.8 \%)$ & $29 / 105(27.6 \%)$ \\
\hline Anticipated data quality & 54 & 9 & 13 & $63 / 76(82.9 \%)$ & $29 / 105(27.6 \%)$ \\
\hline Anticipated robustness of analysis & 29 & 16 & 13 & $45 / 58(77.6 \%)$ & $47 / 105(44.8 \%)$ \\
\hline 1 & 2 & 3 & 4 & 5 & 6 \\
\hline \multirow[t]{2}{*}{$\begin{array}{l}7 \text { respondents }{ }^{*} 15 \text { products } \\
\text { Total } N=105\end{array}$} & Yes & $\begin{array}{l}\text { Maybe/some aspects } \\
\text { of PB-MEA }\end{array}$ & No & $\begin{array}{l}N \text { and percentage of } \\
\text { responses Yes or } \\
\text { Maybe }\end{array}$ & $\begin{array}{l}\text { Not applicable or } \\
\text { not assessed }\end{array}$ \\
\hline & $N$ & $N$ & $N$ & $N / N(\%)$ & $N / N(\%)$ \\
\hline $\begin{array}{l}\text { Has a PB-MEA for this specific product/ } \\
\text { indication been put in place or if not, } \\
\text { what have been the barriers? }\end{array}$ & 1 & 2 & 62 & $3 / 65(4.6 \%)$ & $40 / 105(38.1 \%$ \\
\hline $\begin{array}{l}\text { Would a PB-MEA have been preferred if } \\
\text { these barriers were overcome? Why? }\end{array}$ & 11 & 13 & 34 & $24 / 58(41.4 \%)$ & $47 / 105(44.8 \%)$ \\
\hline $\begin{array}{l}\text { Would cooperation with other CAPRs } \\
\text { have helped address the uncertainties } \\
\text { that warrant a PB-MEA and, if yes, what } \\
\text { kind of cooperation? }\end{array}$ & 31 & 6 & 31 & $37 / 68$ (54.4\%) & $37 / 105$ (35.2\%) \\
\hline
\end{tabular}

The upper panel shows responses to questions about the appropriateness and relevance of various domains of the regulatory postauthorization requirements of fifteen product-indication pairs. The lower panel shows responses to the first (categorizing) part of the questions about a PB-MEA for each individual product-indication pair; answers to the elaboration questions are shown in Table 4. Note that the numbers shown in column 5 are the sum of columns 2 and 3 , and the denominator of the percentages shown in column 5 is exclusive of cases that were deemed "not applicable or not assessed" (as shown in column 6).

CAPR, Competent authorities for pricing and reimbursement; PB-MEAs, performance-based managed entry agreements.

the manufacturer to gather additional data or help with price (re) negotiations.

\section{Discussion}

This work offers insights from the perspective of a limited number of CAPRs into the opportunities and obstacles of PB-MEAs for novel drugs and into potential synergies between regulatory postauthorization requirements and the data required by CAPRs.

Our findings revealed that PB-MEAs are not widely used in the countries that were represented in the survey of CAPRs, even in the context of recent and complex products that were part of our cohort (Table 2); this is in line with findings from the OECD report (2). Setting aside self-evident organizational reasons, such as hospital-only drugs that can be outside legal remit, CAPR assessors identified three broad categories of obstacles to implementing PB-MEAs (Table 4, part 2):
(1) In a situation where the dominant issue is price or (uncertainty about) budget impact, rather than uncertainty about clinical effectiveness, it is felt that the collection of more clinical data would not address the issue.

(2) For several product-indication pairs, payers stated that price competition or other types of financial agreements (e.g., budget caps, price volume agreements) are more appropriate ways to manage their risk. Examples include products entering a competitive field (e.g., "3rd in class") or where the (anticipated) introduction of biosimilars or other new entrants is expected to strengthen the competitive market. Other examples are products with several indications; where indicationbased pricing is not feasible, a PB-MEA is not practical for a second/third indication though it might have been deemed justified for the first indication.

(3) The reviewers repeatedly cited the complexity in structuring and executing PB-MEAs, in some cases referring to past 
Table 3. Summary of favorable responses to individual product-indication pairs.

\begin{tabular}{|c|c|c|c|c|c|c|c|c|c|c|c|c|c|c|c|c|c|}
\hline \multirow{2}{*}{$\begin{array}{l}\text { Responses } \\
\text { from } 7 \text { CAPR } \\
\text { reviewers: }\end{array}$} & \multicolumn{17}{|c|}{ Likely + Partly appropriate and relevant as is (combined) } \\
\hline & Osimertinib & Sacubitril & Nivolumab & Pembrolizumab & Eltrombopag & Lenalidomide & Tisagenlecleucel & Bavacizumab & Cemiplimab & Axicabtagene & Brentuximab & Adalimumab & Larotrectinib & Ranibizumab & Certolizumab & MEAN MIN & N MAX \\
\hline $\begin{array}{l}\text { Patient } \\
\text { population } \\
\text { (including } \\
\text { subgroup } \\
\text { information) }\end{array}$ & 6 & 7 & 5 & 5 & 5 & 5 & 6 & 4 & 5 & 6 & 4 & 4 & 3 & 3 & 3 & 4.73 & 7 \\
\hline $\begin{array}{l}\text { Intervention } \\
\text { (relevance of } \\
\text { dose, } \\
\text { regimen, } \\
\text { place in } \\
\text { therapeutic } \\
\text { pathway, } \\
\text { setting in the } \\
\text { healthcare } \\
\text { system) }\end{array}$ & 6 & 7 & 6 & 5 & 5 & 5 & 4 & 4 & 4 & 5 & 4 & 4 & 5 & 3 & 3 & 4.73 & 7 \\
\hline $\begin{array}{l}\text { Comparators } \\
\text { (the ability to } \\
\text { draw } \\
\text { conclusions } \\
\text { about } \\
\text { comparative } \\
\text { effectiveness, } \\
\text { direct or } \\
\text { indirect } \\
\text { analyses) }\end{array}$ & 5 & 6 & 5 & 5 & 4 & 4 & 1 & 4 & 0 & 1 & 4 & 3 & 1 & 3 & 2 & 3.20 & 6 \\
\hline $\begin{array}{l}\text { Outcomes } \\
\text { (relevance of } \\
\text { end point(s) } \\
\text { to patients, } \\
\text { CAPR) }\end{array}$ & 5 & 6 & 4 & 5 & 5 & 5 & 5 & 4 & 6 & 4 & 4 & 4 & 4 & 3 & 3 & 4.53 & 6 \\
\hline $\begin{array}{l}\text { Time horizon } \\
\text { (duration of } \\
\text { the } \\
\text { observation } \\
\text { period) }\end{array}$ & 6 & 6 & 6 & 5 & 4 & 5 & 5 & 4 & 6 & 4 & 3 & 2 & 4 & 3 & 3 & 4.42 & 6 \\
\hline $\begin{array}{l}\text { Anticipated } \\
\text { data quality }\end{array}$ & 7 & 5 & 6 & 5 & 5 & 5 & 5 & 4 & 3 & 3 & 4 & 3 & 2 & 3 & 3 & 4.22 & 7 \\
\hline $\begin{array}{l}\text { Anticipated } \\
\text { robustness of } \\
\text { analysis }\end{array}$ & 6 & 1 & 5 & 4 & 4 & 3 & 4 & 3 & 3 & 2 & 2 & 2 & 2 & 2 & 2 & 3.01 & 6 \\
\hline Total & 41 & 38 & 37 & 34 & 32 & 32 & 30 & 27 & 27 & 25 & 25 & 22 & 21 & 20 & 19 & & \\
\hline
\end{tabular}


Yes and Maybe/some aspects of PB-MEA (combined)

Responses Tisagenlecleucel Axicabtagene Larotrectinib Cemiplimab Lenalidomide Adalimumab Nivolumab Osimertinib Sacubitril Bavacizumab Brentuximab Eltrombopag Pembrolizumab Certolizumab Ranibizumab MEAN MIN MAX from 7 CAPR

from 7 CAPR

reviewers:

Has a

PB-MEA for

this specific
product/

product/

indication
been put in

place or if

not, what

have been

the barriers?

Would a
PB-MEA have

PB-MEA
been

preferred if

these barriers

were

overcome?

Why?

Would

cooperation
with other

CAPRs have

helped

address the

uncertainties

that warrant

a PB-MEA

and, if yes,

what kind of
cooperation?

Total 12

9

7

5

3

3

2

$2 \quad 1$

1

0

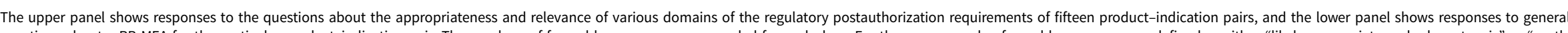

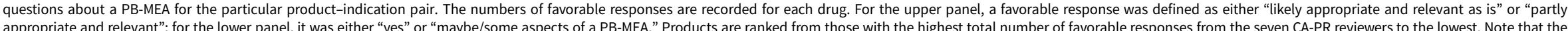
appropriate and relevan", for the lower panel, it was either "yes" or "maybe/some aspects of a PB-MEA." Products are ranked from those with the highest tolal aum

CAPR, Competent athoities for pricing and reimbursem and PB MEAs, performance based manaed enty ageements. 
Table 4. Qualitative analysis of responses to the CAPR survey

1. Questions and answers on the individual domains of the EMA's postauthorization requirements

Reasons stated for not providing information on one or more questions or replying "nonapplicable":

- Insufficient information/detail provided by the EMA in the public domain;

- Inadequate description of postauthorization studies;

- Cannot judge in the absence of study protocol(s).

Reasons stated why the patient population (including subgroup information) is inadequate/inappropriate/not relevant:

- The product is studied in the first line, and expected use in our jurisdiction will be second or third line.

Reasons stated why the intervention (dose, regimen, place in therapeutic pathway, setting in the healthcare system) is inadequate/inappropriate/not relevant: - Inadequate description of complex dose regimen.

Reasons stated why the comparator (the ability to draw conclusions about comparative effectiveness, direct or indirect analyses) is inadequate/inappropriate/ not relevant:

- The lack of control arm/only single-arm study;

- Studies would not allow for indirect comparisons;

- Nonpharmaceutical treatments (such as physiotherapy and surgery) that can be validated alternatives to be used as a comparator, have not been considered.

Reasons stated why the outcome (relevance of end point(s) to patients, CAPR) is inadequate/inappropriate/not relevant:

- The lack of information on the quality of life;

- Required postauthorization studies were only for safety, not enough information expected on efficacy/effectiveness;

- The end point is not a validated surrogate for overall survival.

Reasons stated why the time horizon (duration of observation period) is inadequate/inappropriate/not relevant:

- Longer-term data would be needed to capture relevant end points.

Reasons stated why the anticipated data quality is inadequate/inappropriate/not relevant:

- Only observational data (i.e., nonrandomized study);

- Required postauthorization study too small.

Reasons stated why the anticipated robustness of analysis is inadequate/inappropriate/not relevant:

- Observational study only;

- No control group.

2. Questions and answers on the implementation, desirability of a PB-MEA, and cooperation with other CAPRs

Reasons stated for not providing information on PB-MEAs or replying "nonapplicable":

- An MEA (but not necessarily a PB-MEA) is in place for this product, but circumstances are confidential.

- The product is still under evaluation.

- The product will not be reimbursed in this jurisdiction/negative reimbursement decision.

- To date, no submission for reimbursement has been received for this product.

Reasons stated why a PB-MEA is not in place/is not preferred for this product/what are the barriers (grouped by overarching themes):

- Outside of CAPR's remit:

- For this product, hospitals negotiate the price with the manufacturer, and health insurance companies negotiate the price on behalf of the insured citizens with the hospitals.

- This is a hospital-only product, and therefore, is not in our remit.

- Price/budget impact is the main parameter to manage:

- The only problem is price and budget impact. This is well understood, but the collection of more data cannot solve this problem.

- Effectiveness is not in question, financial uncertainty is the main barrier, but a PB-MEA would not address this.

- If the budget impact is the main problem, payers will use other types of financial agreements (e.g., price volume with several discount levels or a maximum budget) that are far easier to implement in order to limit the risk.

- Pricing that can match demonstrated value is always preferable.

- Value is not proven, and, therefore, the product is not recommended for reimbursement.

- Other types of MEAs or strategies preferred (e.g., budget cap; price volume agreements):

- The product enters a competitive field. We would not consider a PB-MEA, price competition is a more obvious way to reduce the risk for payers.

- This product is at least 3rd in class, alternatives available.

- The (expected) introduction of biosimilars strengthens the competitive market.

- The well-known product is already used for several indications. The new indication is not assessed but enters the insurance coverage without explicit reimbursement decision.

- Product is already reimbursed; we received no application for this indication.

- The product has several indications. Indication-based pricing is not feasible now.

- PB-MEA not suitable for a second/third indication but might have been suitable for first indication of this product.

- At the price we negotiated, cost-effectiveness seemed robust; if price were higher, perhaps a PB-MEA would have been helpful.

- Complexity in structuring/executing PB-MEA:

- Main barriers for PB-MEAs are the complexity of the agreement and execution/implementation, data collection, etc.

- Results from PB-MEAs are often late or of poor quality and inconclusive.

- End point(s) rather difficult to use for a PB-MEA.

- Different body responsible for price negotiation and for PB-MEAs, organizational complexity too high.

Reasons stated why a PB-MEA is in place/would have been preferred for this product if these barriers were overcome:

- A PB-MEA would have been justified because of the uncertain long-term efficacy.

- We consider MEAs post cost-effectiveness and price negotiation to manage entry of the product for some subgroups only which we believe to be cost-effective, rather than the full indication population.

- Greatest uncertainty lies with benefit (efficacy) which a PB-MEA could address.

- A PB-MEA might only be an option if there is a high price, significant budget impact and a very clearly defined uncertainty regarding the (comparative) effectiveness of the medicine in an indication without competition.

Reasons stated why cooperation with other CAPRs would have helped address the uncertainties that warrant a PB-MEA/what kind of cooperation:

- Cooperation could help jointly incentivizing the manufacturer to gather the data or by collaborating on (inter)national registries.

- We might learn faster about the best way to use the medicine.

- Cooperation might have been helpful for purposes other than a PB-MEA, for example, price re-negotiations.

- An option could have been early cooperation that could have resulted in the company including a European-wide relevant comparator group in the pivotal study. 
- Collaboration on additional data collection combined with information on how pricing is organized.

- Collaboration may be helpful for patients with less common mutations. If payers could collaborate and all indicate that additional research regarding these mutations is necessary, this may provide stronger incentives for the manufacturer to perform these studies.

Reasons stated why cooperation with other CAPRs would not have helped address the uncertainties that warrant a PB-MEA:

- Not clear that other CAPRs would have the information to reduce uncertainty.

- Cooperation would not have convinced us to enter a PB-MEA.

- Standardization of outcomes across jurisdictions would not be easy.

Part 1 lists questions and answers on the individual domains of the EMA's postauthorization requirements, Part 2 lists questions and answers on the implementation or desirability of a PB-MEA and on cooperation with other CAPRs.

CAPR, Competent authorities for pricing and reimbursement; PB-MEAs, performance-based managed entry agreements.

experiences with PB-MEAs. It was felt that results from PB-MEAs are often late or inconclusive; for some productindication pairs, the clinical end point(s) were deemed too difficult to adjudicate for use for a PB-MEA.

In contrast to the very small number of PB-MEAs implemented, the reviewers were more positive about the potential value of PB-MEAs for these products and about cooperation with other organizations-if only the conditions were right (Table 2, lower panel).

The perceived potential value of a PB-MEA varied widely from one product to the next, as can be observed in Table 3 . This is not unexpected, given that we deliberately chose a highly heterogeneous sample of product-indication pairs, comprising treatments for very diverse rare and common conditions; small molecule, biologic, and advanced therapies; and a subset of conditionally authorized products. Hence, our analysis (Table 4, part 2) also provides insights into the types of products and indications that may motivate CAPRs to overcome the barriers associated with the implementation of PB-MEAs. Drawing on the responses provided, we submit that the type of product-indication pair for which a PB-MEA could be considered is one that ticks all (or most of) the boxes below:

(1) a new treatment, promising in terms of relieving an unmet need;

(2) an identified uncertainty regarding its (comparative) effectiveness or an unknown such as where the authorization is broader than the clinical data submitted for market authorization;

(3) an indication where patient relevant end points or surrogate outcomes can be captured easily in usual medical practice;

(4) a high price, uncertain budget impact or uncertain value;

(5) a first authorized indication;

(6) an indication without (anticipated) competition.

Moreover, if these conditions are met, collaboration across CAPRs and regulators may facilitate the implementation of PB-MEAs and other aspects of pricing and reimbursement negotiations (Table 4, part 2).

In regard to the regulator-CAPR collaboration, our results show that, in the eyes of the CAPR reviewers, the information currently available in the public domain on postauthorization requirements is often insufficient or not sufficiently granular to inform the implementation of PB-MEAs. Table 2 (upper panel, column 6) shows that reviewers deemed the information on specific aspects of the postauthorization requirements "not applicable or not assessed" in around a quarter of instances. The most frequently cited reasons were inadequate description of postauthorization studies and inaccessibility of study protocol(s) in the public domain. Although these findings are strictly applicable only to the information provided by the EMA, they may have relevance for information shared by regulators in other jurisdictions.

Note that the "not applicable or not assessed" responses are agnostic about the (in-)adequacy of the underlying regulatory postauthorization requirements. We, therefore, present results excluding this category of responses to separate the issues of insufficient information versus genuine inadequacy of postauthorization requirements (such as "required post-authorization study too small").

Yet, where they were able to assess regulatory postauthorization requirements, the CAPR reviewers rated regulatory postauthorization requirements "likely or at least partly appropriate and relevant" for most of the domains studied (Table 2, upper panel, column 5), the only exception being the comparator domain. The latter finding likely reflects the different roles of regulators and CAPRs in regard to the assessment of comparative efficacy/effectiveness information (6).

Acknowledging that simply summing up the favorable responses about the different domains of the regulatory postauthorization requirements is a crude tool, we still argue that the wide range of favorable responses for individual product-indication pairs (Table 3, upper panel) indicates that CAPRs considered the study sample of product-indication pairs highly heterogeneous; only for some drugs were the postauthorization requirements considered appropriate to reduce uncertainty about the drug's performance and to help design and execute a successful PB-MEA.

These mixed results show that, on the one hand, the current level of the perceived utility of the regulatory postauthorization requirements may be insufficient to change the use of PB-MEAs.

On the other hand, our findings support the notion that the differences in information needs during the postauthorization period between regulators and CAPRs are not insurmountable. This agrees with the experience gained in the context of parallel scientific advice during the preauthorization clinical development phase. Tafuri et al. (4) analyzed the learnings from parallel scientific advice procedures, which allow manufacturers to receive simultaneous feedback from both EU regulators and CAPR bodies on their development plans for new medicines. They found commonality, in terms of evidence requirements between regulators and participating CAPR bodies, as well as among CAPR bodies, on most aspects of clinical development. Of note, for that sample, the level of agreement between CAPR bodies and the regulators was high for the domains patient population, end points and other study design characteristics but much lower for the comparator domain, a finding that closely mirrors our own results presented in Table 2 (upper panel).

The need for better alignment on "post-launch evidence generation (PLEG)" between different decision makers and manufacturers has been recognized and parallel scientific advice procedures with multiple decision makers have been advocated 
as a promising route to improve the design and research efficiency of PLEG studies $(7 ; 8)$.

Parallel scientific advice was deemed successful in the preauthorization space $(4 ; 9)$, and, therefore, we would expect a similar degree of success for parallel scientific advice on PLEG studies.

There are several limitations with our study: our group of CAPR reviewers comprises a convenience sample from wealthier countries, not necessarily representative of other public, national, or private healthcare payers or CAPR bodies, and that from lowerincome countries. Some CAPRs with extensive experience with PB-MEAs are not represented in our study. This may limit the applicability and relevance of our conclusions to other healthcare settings. Yet, our observations are at least broadly in line with the positions and views expressed by other decision makers external to our study team (10).

Another limitation is grounded in the nature of our survey, which was not based on multiple choice-style questions but invited free text comments from CAPR reviewers. A variation in granularity and scope of responses was expected and precluded more in-depth quantitative analyses than what is presented in Tables 2 and 3. The categorizations used in our template (Table 2) are necessarily an oversimplification based on individual judgment and, therefore, cannot be considered as quantitative measurements. On the other hand, the open-ended questions allowed for more insights into the reasoning of reviewers, as presented in Table 4.

Last, in some instances, the CAPR reviewers were unable to share their views on a particular product-indication pair due to the confidentiality of an arrangement in place between their organizations and pharmaceutical companies. For example, for Australia, only PSDs could be used (5). However, this limited the analysis in only a handful of cases.

There are several major strengths of this work. First, this was, to our knowledge, the first attempt to explore the opportunities and obstacles of regulator-CAPR collaboration to facilitate PB-MEAs. Second, we selected a cohort of recently authorized product-indication pairs that come with a range of challenges for pricing and reimbursement agreements (2); our sample included orphan and cancer drugs, complex biologics and gene therapies, and conditionally authorized products. Given the evolving nature of products expected in the future (11), our study cohort seems highly predictive of future challenges for both CAPRs and drug manufacturers. Third, the CAPR reviewers in our study had first-hand experience with pricing and reimbursement negotiations and were familiar with almost all product-indication pairs, adding practical relevance to our findings.

Using our findings and the recent OECD report (2) on PB-MEAs as a starting point, what further concrete steps could be taken by CAPRs, regulators, and manufacturers to improve the effectiveness of PB-MEAs in achieving their stated goals and to support regulators in delivering on their mission to monitor drug performance?

We suggest that there are at least three levels on which to progress:

First, establishing a conceptual framework for prioritizing products and indications for which PB-MEAs could support timely access for patients. We have proposed above a short list of "selection criteria" that might serve as a basis for future discussions between manufacturers and CAPRs to systematically and prospectively identify those product-indication pairs for which all stakeholders could be motivated to address the technical hurdles associated with the implementation of such complex arrangements.
Second, manufacturers, regulatory agencies, and CAPRs should work together to discuss the evidence-generation strategy for cases where PB-MEAs are deemed to be an option before they enter the market. Parallel scientific advice for PLEG has been proposed and described in some detail (7) as a promising avenue to achieve agreement on the most economic strategy to satisfy both regulators' information needs and to ensure that PB-MEAs can be successful in achieving their goals. The anticipated benefits of a successful PB-MEA for all stakeholders would likely justify any additional resources required to conduct parallel scientific advice procedures.

Third, the level of transparency and information sharing around PLEG activities needs to be raised in more than one way. Our findings show that the information currently provided by the EMA (and perhaps by other regulatory agencies) may be insufficient to inform CAPRs in their efforts to implement PB-MEAs. This could be remedied by reaching either general or product-specific agreements on what additional information, such as study protocols, should be made public.

However, there is another frontier where transparency needs to improve: the OECD report (2) highlighted that results of MEA agreements, including those of PB-MEAs, are often confidential, and information generated under the agreements is not shared with other CAPRs and third parties to advance knowledge. We believe that secrecy about the clinical, though not the financial, results of postauthorization data generation plans is not only a missed opportunity for progressing our understanding of drug actions but also violates an ethical imperative to share what can be considered a common good. The past years have seen tremendous progress in transparency around preauthorization studies. Now is the time to do the same for information generated post launch; the need for detailed and precise documentation in protocols and reports to enable the interpretation of (observational) postauthorization studies has been repeatedly elaborated $(12 ; 13)$.

In conclusion, PB-MEAs are not often the preferred option for CAPRs to deal with uncertainty. Our study identified a set of hurdles but also conditions under which a PB-MEA might be worth considering. It remains to be seen whether this may help CAPRs to identify those products where PB-MEAs would help address uncertainty gaps at the time of launch to an extent that would justify the implementation of such complex agreements. We are aware of the many issues of implementing PB-MEAs, including legal hurdles, unwillingness of manufacturers to enter such agreements, differences in the perception of benefit between payers, and administrative workload. However, collaboration across silos and more transparency around PLEG activities could help overcome some of the barriers associated with the data generation part of PB-MEAs.

\section{Disclaimer}

The views expressed in this article are the personal views of the author(s) and may not be understood or quoted as being made on behalf of or reflecting the position of the regulatory agency/agencies or organizations with which the author(s) is/are employed/affiliated.

Acknowledgments. Orla Maguire, National Centre for Pharmacoeconomics (NCPE Ireland), Dublin, Ireland and Niamh Carey, National Centre for Pharmacoeconomics (NCPE Ireland), Dublin, Ireland and Trinity College Dublin, Dublin, Ireland, assisted in the descriptive analysis of the products as well as the review of the manuscript.

Funding. This research received no specific grant from any funding agency, commercial, or not-for-profit sectors. 
Conflicts of Interest. There are no conflicts of interest.

\section{References}

1. Hwang TJ, Ross JS, Vokinger KN, Kesselheim AS. Association between FDA and EMA expedited approval programs and therapeutic value of new medicines: Retrospective cohort study. BMJ. 2020;371:m3434. doi:10.1136/ bmj.m3434. PMID: 33028575; PMCID: PMC7537471.

2. Wenzl M, Chapman S. Performance-based managed entry agreements for new medicines. In: OECD countries and EU member states: How they work and possible improvements going forward. OECD Health Working Papers, No. 115. Paris: OECD Publishing; 2019. doi:10.1787/6e5e4c0f-en.

3. European Medicines Agency (EMA). Conditional marketing authorisation. Available from: https://www.ema.europa.eu/en/human-regulatory/ marketing-authorisation/conditional-marketing-authorisation.

4. Tafuri G, Pagnini M, Moseley J, Massari M, Petavy F, Behring A, et al. How aligned are the perspectives of EU regulators and HTA bodies? A comparative analysis of regulatory-HTA parallel scientific advice. $\mathrm{Br} \mathrm{J}$ Clin Pharmacol. 2016;82:965-73. doi:10.1111/bcp.13023. Epub 2016/07/ 01. PMID: 27245362; PMCID: PMC5137821.

5. Australian Government Department of Health. Public summary documents. Available from: https://www.pbs.gov.au/pbs/industry/listing/elements/pbac-meetings/psd.

6. Kleijnen S, George E, Goulden S, d'Andon A, Vitré P, Osińska B, et al. Relative effectiveness assessment of pharmaceuticals: Similarities and differences in 29 jurisdictions. Value Health. 2012;15:954-60. doi:10.1016/ j.jval.2012.04.010. Epub 2012/06/20. PMID: 22999147.

7. Moseley J, Vamvakas S, Berntgen M, Cave A, Kurz X, Arlett P, et al. Regulatory and health technology assessment advice on postlicensing and postlaunch evidence generation is a foundation for lifecycle data collection for medicines. Br J Clin Pharmacol. 2020;86:1034-51. doi:10.1111/ bcp.14279. Epub 2020/04/24. PMID: 32162368; PMCID: PMC7256124.

8. Facey KM, Rannanheimo P, Batchelor L, Borchardt M, de Cock J. Real-world evidence to support payer/HTA decisions about highly innovative technologies in the EU-actions for stakeholders. Int $J$ Technol Assess Health Care. 2020; 1-10. doi:10.1017/S026646232000063X. Epub ahead of print. PMID: 32878663.

9. Tafuri G, Lucas I, Estevão S, Moseley J, d'Andon A, Bruehl H, et al. The impact of parallel regulatory-health technology assessment scientific advice on clinical development. Assessing the uptake of regulatory and health technology assessment recommendations. $\mathrm{Br} J$ Clin Pharmacol. 2018;84:1013-19. doi:10.1111/bcp.13524. Epub 2018/03/05. PMID: 29370449; PMCID: PMC5903262.

10. Cooper S, Bouvy JC, Baker L, Maignen F, Jonsson P, Clark P, et al. How should we assess the clinical and cost effectiveness of histology independent cancer drugs? BMJ. 2020;368:16435. doi:10.1136/bmj.16435. PMID: 31896539.

11. Eichler HG, Pignatti F, Schwarzer-Daum B, Hidalgo-Simon A, Eichler I, Arlett P, et al. Randomised controlled trials versus real world evidence: Neither magic nor myth. Clinical Pharmacology and Therapeutics. 2020. doi:10.1002/cpt.2083. Epub ahead of print. PMID: 33063841.

12. European Medicines Agency (EMA). Big data. Available from: https:// www.ema.europa.eu/en/about-us/how-we-work/big-data\#hma/ema-big-datasteering-group-section.

13. Wang SV, Pinheiro S, Hua W, Arlett P, Uyama Y, Berlin JA, et al. STaRT-RWE: Structured template for planning and reporting on the implementation of real-world evidence studies. $\mathrm{Br}$ Med J. 2021;372: $\mathrm{m} 4856$. 